\title{
Comparación entre las normas de desempeño térmico edilício de Argentina y Brasil
}

\author{
Comparação entre normas de desempenho térmico de \\ edificações da Argentina e do Brasil
}

\section{Gabriela Reus Netto Jorge Daniel Czajkowski}

\section{Resumen}



1 objetivo de este estudio es realizar una revisión de la normativa de desempeño térmico destinada a edificios de viviendas, vigente en Argentina y Brasil. Se busca detectar las coincidencias y divergencias más significativas, así como la trayectoria y dificultades para su implementación, con la finalidad de proponer acciones y mejoras. Se asume que con un programa de gestión adecuado que incluya participación social y profesional, se podrá aumentar el nivel de compromiso ambiental, la aplicación efectiva de normas, como un requisito para modificar la cultura vigente en la edificación. El análisis se basó en el examen de documentos normativos y legales sobre el desempeño térmico edilicio, además del material libre disponible. De Argentina, se analizó el conjunto de las normas IRAM enumeradas en el Decreto 1.030/10, que reglamenta la Ley 13.059/03 y el código de edificaciones de Rosario. Para Brasil se estudió la NBR 15220/08 y la 15575/13. Se contrastaron las normas citadas y se encontró que en cuanto a valores exigidos en transmitancia térmica para muros, la IRAM es 2 veces más exigente que la NBR en verano y 3,5 más, en invierno. Finalmente se proponen recomendaciones que contribuyan al mejoramiento en la aplicación del desempeño térmico del sector residencial.

Palablas-claves: Normativa. Brasil. Argentina. Desempeño térmico edilicio. Edificaciones residenciales.

\section{Resumo}

O objetivo deste estudo é realizar uma revisão da normativa de desempenho térmico destinada a edifícios residenciais em vigor na Argentina e no Brasil. Busca-se detectar as coincidências e divergências mais significativas, assim como a trajetória e as dificuldades para sua implementação. Assume-se que com um programa de gestão adequado, que inclua a participação social e profissional, se poderá aumentar o nível de compromisso ambiental e a aplicação efetiva das normas, como um requisito para modificar a cultura vigente na edificação. A análise se baseou no estudo de documentos normativos e legais sobre desempenho térmico de edificações, além do material livre disponível. Da Argentina se examinou o conjunto das normas

Gabriela Reus Netto Universidad Nacional de La Plata Buenos Aires - Argentina

Jorge Daniel Czajkowski Universidad Nacional de La Plata Buenos Aires - Argentina

Recebido em 14/04/15 Aceito em 17/07/15
IRAM enumeradas no Decreto 1.030/2010, que regula a Lei 13.059/2003 e o código de obras de Rosário. Para Brasil se estudaram a NBR 15220/2008 e a NBR 15575/2013. Compararam-se as normas citadas e se encontrou que, quanto a valores estabelecidos para a transmitância térmica das paredes, a norma IRAM é duas vezes mais exigente que a NBR no verão, e 3,5 vezes mais no inverno. Finalmente se propõem recomendações que contribuam com a melhoria da aplicação do desempenho térmico do setor residencial.

Palavras-chaves: Normas. Brasil. Argentina. Desempenho térmico de edifícios. Edificações residenciais. 


\section{Introducción}

Favorecida por los caudalosos ríos presentes en Brasil, en 2011 las hidroeléctricas representaban más del $80 \%$ de la matriz eléctrica nacional, según datos del Instituto Brasilero de Geografía y Estadística - IBGE ${ }^{1}$. La fuerte sequía que afectó a Brasil, principalmente San Pablo a fines del 2014, juntamente con los consumos records en energía eléctrica debido a las elevadas temperaturas y demás factores administrativos de las distribuidoras de energía en el país, tuvieron efectos en el aumento de las tasas de los habitantes que consumen más agua con un incremento que varía de $21,25 \%$ a $39,49 \%$ en las tarifas de energía eléctrica residencial ${ }^{2}$.

En Argentina, las energías fósiles representan el $89 \%$ de la matriz energética nacional (EVANS; SCHILLER, 2010). La escasa disponibilidad de recursos y el elevado consumo es una problemática que viene incrementándose. En el año de 2013, según Jorge Oviedo ${ }^{3}$ los gastos de importación de gas natural, licuado y combustibles llegaron a 12.800 millones de dólares y solamente en el primer semestre de 2014 se importaron aproximadamente cuatro veces más energía que en todo el año de 2013

En la búsqueda de disminuir el consumo de recursos energéticos para el acondicionamiento térmico y lumínico, mejorar el confort interno de los ambientes, garantizar el desempeño térmico mínimo edilicio y auxiliar en la construcción, se establecen pautas, recomendaciones y normas.

Alemania, Canadá, Italia y España han sido pioneros en la regulación relativa el desempeño térmico de las edificaciones. En Argentina, el escenario del desempeño térmico de edificios cuenta con un conjunto de normativas que vienen siendo estudiadas y actualizadas desde el año de 1964 hasta el presente. A pesar de ser obligatorias por la Ley en la Provincia de Buenos Aires y la Ciudad Autónoma de Buenos Aires desde el año 2003 y 2012, respectivamente; no se hace efectivo su cumplimiento.

En Brasil, el Grupo de Conforto Ambiental e Eficiência Energética de la ANTAC, viene desde 1988, trabajando en la búsqueda de elaborar un

${ }^{1}$ Sitio web del IBGE. Principales Estadísticas de la Energía Mundial - IEA de 2008. Consultado: 16/04/2014.

${ }^{2}$ Julia Borba para Folha de São Paulo -

http: //www1.folha.uol.com.br/mercado/2015/02/1584514aneel-aprova-reajuste-de-sete-distribuidoras-aumento-chega-a457.shtml Consultado: 5/2/2015

3 Jorge Oviedo para La Nación -

http: / / www.lanacion.com.ar/1714152-energia-en-un-mesimportaron-de-uruguay-mas-que-en-todo-2013 Consultado: $6 / 8 / 2014$ proyecto de normativa de confort para ser aplicada en todo Brasil (RORIZ; GHISI; LAMBERTS, 1999). Como resultado del "I Encuentro Nacional sobre Norma en Uso Racional de Energía y Confort Ambiental en Edificaciones", realizado en 1991 surgieron algunas propuestas.

En Florianópolis, en 2005, se instauró a nivel informativo la primera norma destinada a garantizar un nivel mínimo de desempeño térmico de viviendas de interés social. En 2008, con carácter obligatorio, se implementó una segunda norma para garantizar el desempeño edilicio de obras en general, inclusive térmico y energético y en 2013 entró oficialmente en vigencia.

Aunque se verifique el trabajo por parte del Estado en promover y facilitar la adaptación a las normas, su cumplimiento no se ve reflejado en la totalidad del territorio argentino y brasilero (SILVA et al., 2011).

Es evidente una mejora en la calidad de las edificaciones pero es necesario llegar a una idea de confort y ahorro energético durante el ciclo de vida del edificio (GONÇALVES; DUARTE, 2006). A partir de estudios y experiencias desarrolladas en Brasil se puede observar que a pesar de que existen viviendas que cumplen con los valores determinados en las normas, aún no se obtiene confort en los ambientes internos (GRIGOLETTI; LINCK, 2014). Según Gonçalves y Duarte (2006) la teoría no concuerda con la opinión de los usuarios, así como las quejas son parecidas para realidades climáticas diferentes. De acuerdo a las auditorías realizadas en Argentina (CZAJKOWSKI et al., 2012) el elevado consumo de gas natural utilizado en invierno para la calefacción no alcanza a satisfacer las necesidades de confort de los residentes es decir, tanto los sistemas de climatización como la capacidad de las viviendas de conservar el calor es baja (BERARDI et al., 2014).

Los diversos obstáculos que impiden la implementación intensiva de dichas normas, generan consecuentemente viviendas que por lo determinante del aspecto económico en la etapa de construcción, no presentan la debida adaptación a los diversos climas, al sitio, a las necesidades del usuario, y se nota una poca preocupación en preservar la vida útil del edificio. En estudios realizados en Argentina y Brasil (GALLIPOLITI et al., 2012; DI BERNARDO; FILIPPÍN; PIPA, 2011), han encontrado casos que previo a la entrega de la obra de presentan patologías relacionadas con el sellado de las envolventes y la condensación, es por ello que durante la etapa de

106 Reus Netto, G.; Czajkowski, J. D. 
uso el usuario utiliza continuamente artefactos para acondicionamiento térmico (GRIGOLETTI; LINCK, 2014; CZAJKOWSKI et al., 2012), factor que por ejemplo justifica la creciente demanda eléctrica en Argentina, la consecuente importación y aumento de la deuda externa (GIL, 2014).

En este contexto, la importancia del tema de investigación corresponde:

(a) comparar las normativas pertinentes;

(b) comprender sus principales coincidencias y diferencias; $y$

(c) proponer recomendaciones que contribuyan a la aplicación de la regulación en la totalidad de Brasil y Argentina.

Se pretende alcanzar la mejora del desempeño edilicio, especialmente en el ámbito del confort térmico, lo que permitirá mejorar la calidad de vida del usuario y ahorrar recursos energéticos, minimizando el impacto ambiental.

\section{Metodología}

Este trabajo consistió primero, examinar el conjunto de las Normas Argentinas IRAM destinadas a garantizar las "condiciones de habitabilidad higrotérmica, de higiene, y de salubridad", enumeradas en el Decreto 1.030 de 02 de julio de 2010 (ARGENTINA, 2010), que reglamenta la Ley $\mathrm{n}^{\circ}$ 13.059/03 (ARGENTINA, 2003) de la provincia de Buenos Aires. Asimismo, estudiar las normas IRAM 11659-1/2 (INSTITUTO..., 2004, 2007) y 11.900 (INSTITUTO, 2007) establecidas en 2010 como documento en estudio y actualmente en vigencia en la ciudad autónoma de Buenos Aires (Documento en estudio de mayo de 2004 - Norma 11659-1 (INSTITUTO..., 2004) / Documento en estudio de 2007-04-12 - Norma 11659-2 (INSTITUTO..., 2007) / Documento en estudio de diciembre de 2009 - Norma 11.900 (INSTITUTO..., 2007)), afectan aproximadamente 18.520.000 habitantes, según el Censo 2010 de INDEC $^{4}$.

En segundo lugar se revisó la normativa brasileña creada para regular la construcción de manera a alcanzar un nivel mínimo de desempeño térmico, NBR 15220 (ABNT, 2008) y NBR 15575 (ABNT, 2013).

En ambos grupos de normativas se identificaron las características climáticas generales, los requisitos y criterios mínimos exigidos en el cumplimiento de las normas y sus procesos de

${ }^{4}$ Disponíble en:

<http://www.censo2010.indec.gov.ar/resultadosdefinitivos_tota lpais.asp>. Accesso en: 05 feb. 2015. implementación en el panorama de la construcción civil. Esto permitió establecer las principales diferencias y similitudes a fin de proponer medidas que representen un perfeccionamiento de las normas, mediante análisis comparativo.

Este trabajo se fundamenta, en el análisis de documentos normativos y legales sobre el desempeño térmico edilicio, además del material libre disponible.

\section{Caso de estudio A: Argentina}

La Ley 13.059/03 (ARGENTINA, 2003), busca definir las condiciones de acondicionamiento térmico exigidas en la construcción de todos los edificios públicos y privados localizados en la Provincia de Buenos Aires. Para eso, establece en el Decreto 1.030/10 (ARGENTINA..., 2010) que toda obra debe cumplir con los siguientes requisitos:

(a) cumplir con los valores de $\mathrm{K}$ admisible - o U $\left(\mathrm{W} / \mathrm{m}^{2} \mathrm{~K}\right)$ - para condiciones de invierno y verano (INSTITUTO..., 1996);

(b) verificación el Riesgo de Condensación superficial e intersticial para paños centrales y singulares (INSTITUTO..., 2000a, 2000b);

(c) comprobar el valor del Coeficiente $\mathrm{G}$ adm para calefacción (INSTITUTO..., 2001);

(d) verificar los valores de calidad térmica $\mathrm{K}$ en vidriados (INSTITUTO..., 2010); y

(e) cumplir con la calidad de infiltración en carpinterías (INSTITUTO..., 2010).

\section{Clima}

El territorio argentino, conforme indica el mapa bioambiental (Figura 1) disponible en la norma IRAM 11603 (INSTITUTO..., 2012) Acondicionamiento térmico de edificios, se encuentra divido en 6 zonas bioambientales, clasificados conforme las características climáticas (Tabla 1).

\section{Requisitos y criterios mínimos}

\section{Transmitancia térmica máxima admisible: K MAX ADM}

La Norma IRAM 11605 (INSTITUTO..., 1996), de diciembre de 1996, titulada "Acondicionamiento térmico de edificios - Condiciones de habitabilidad en edificios - Valores máximos de transmitancia térmica en cerramientos opacos", define tres niveles de confort: A - recomendado, B - Medio y C - Mínimo, para cada uno, de los valores máximos de transmitancia térmica en condiciones 
de verano e invierno. Para cumplir con la norma, la transmitancia térmica de aire a aire de los techos, muros y pisos deberá ser menor o igual a la transmitancia térmica máxima admisible - " $\mathrm{K}$ max adm" correspondiente al nivel B, la verificación debe realizarse simultáneamente en condiciones de verano e invierno, excepto para las zonas bioambientales V y VI, donde solo se exige que se cumpla con la condición de invierno.

Para la condición de verano, los valores máximos admisibles están definidos según se trate de muros o techos y en función de cada zona bioambiental, conforme se verifica en la Tabla 2.

Estos valores de $\mathrm{K}$ están dados para todos elementos cuya superficie exterior presente un coeficiente de absorción de la radiación solar de 0,6 a 0,8 . Para coeficientes menores que 0,6 , se acrecienta al valor de $\mathrm{K}$ el $20 \%$ para muros y $30 \%$ para techos y para los superiores a 0,8 , se disminuye el valor de $\mathrm{K}$ en $15 \%$ y $20 \%$, para muros y techos respectivamente.

Figura 1 - Mapa bioambiental de la República Argentina

Fuente: IRAM 11603 (INSTITUTO..., 2012).

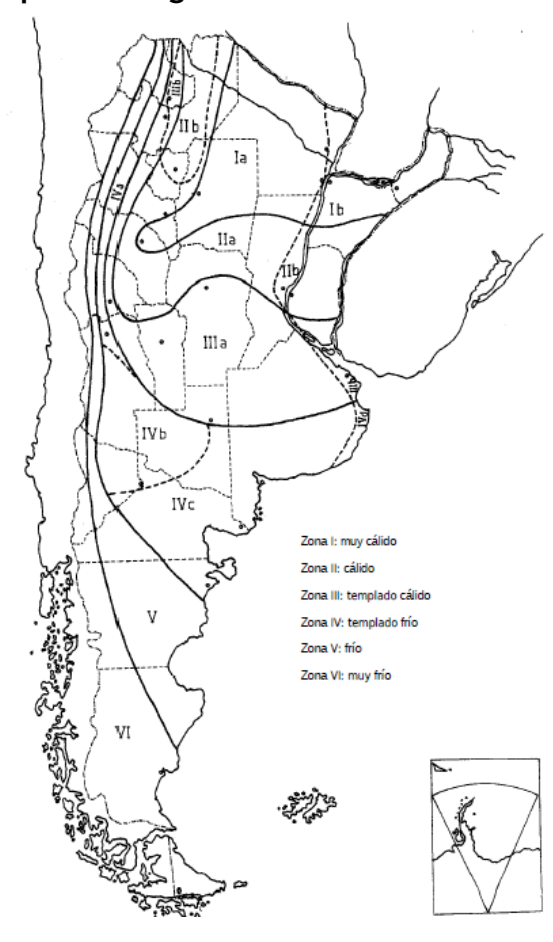

Tabla 1 - Características de las zonas bioclimáticas de Argentina

\begin{tabular}{|c|c|c|c|c|}
\hline \multicolumn{2}{|c|}{ Clasificación } & Zona & $\begin{array}{l}\text { Temperatura efectiva } \\
\text { corregida (TEC) }\end{array}$ & $\begin{array}{l}\text { Amplitud } \\
\text { Térmica }\end{array}$ \\
\hline \multirow{2}{*}{ I - Muy cálida } & A & \multirow{2}{*}{ Centro este del norte del país } & Verano: Mayores a $26,3^{\circ} \mathrm{C}$ & $>$ a $14^{\circ} \mathrm{C}$ \\
\hline & $\mathrm{B}$ & & Invierno: Mayores a $12^{\circ} \mathrm{C}$ & $<\mathrm{a} 14^{\circ} \mathrm{C}$ \\
\hline \multirow{2}{*}{ II - Cálida } & $\mathrm{A}$ & \multirow{2}{*}{$\begin{array}{c}\text { Extensión este - oeste del norte del } \\
\text { país }\end{array}$} & Verano: Mayores a $24^{\circ} \mathrm{C}$ & $>$ a $14^{\circ} \mathrm{C}$ \\
\hline & $\mathrm{B}$ & & Invierno: Entre $8^{\circ} \mathrm{C}$ y $12^{\circ} \mathrm{C}$ & $<\mathrm{a} 14^{\circ} \mathrm{C}$ \\
\hline \multirow{2}{*}{$\begin{array}{c}\text { III - } \\
\text { Templada } \\
\text { cálida }\end{array}$} & A & \multirow[b]{2}{*}{ Predominancia en el centro del país } & Verano: Entre $20^{\circ} \mathrm{C}$ y $26^{\circ} \mathrm{C}$ & $>\mathrm{a} 14^{\circ} \mathrm{C}$ \\
\hline & $\mathrm{B}$ & & Invierno: Entre $8^{\circ} \mathrm{C}$ y $12^{\circ} \mathrm{C}$ & $<$ a $14^{\circ} \mathrm{C}$ \\
\hline \multirow{4}{*}{$\begin{array}{l}\text { IV - } \\
\text { Templada fría }\end{array}$} & $\mathrm{A}$ & \multirow{4}{*}{$\begin{array}{l}\text { Predominancia en el centro sur del } \\
\text { país }\end{array}$} & \multirow{2}{*}{ Verano: $23^{\circ} \mathrm{C}$} & $18^{\circ} \mathrm{C}$ \\
\hline & $\mathrm{B}$ & & & $17^{\circ} \mathrm{C}$ \\
\hline & $\mathrm{C}$ & & \multirow{2}{*}{ Invierno: Entre $4^{\circ} \mathrm{C}$ y $8^{\circ} \mathrm{C}$} & $16^{\circ} \mathrm{C}$ \\
\hline & $\mathrm{D}$ & & & $15^{\circ} \mathrm{C}$ \\
\hline \multirow{2}{*}{ V - Fría } & \multirow{2}{*}{\multicolumn{2}{|c|}{$\begin{array}{l}\text { Extensión norte - sur de la cordillera y la } \\
\text { región central de la Patagonia }\end{array}$}} & Verano: $16^{\circ} \mathrm{C}$ & \\
\hline & & & Invierno: $4^{\circ} \mathrm{C}$ & \\
\hline \multirow{2}{*}{ VI - Muy fría } & \multirow{2}{*}{\multicolumn{2}{|c|}{$\begin{array}{l}\text { Altas cumbres de la Cordillera de los } \\
\text { Andes y el extremo Sur de la Patagonia }\end{array}$}} & Verano: Menores a $12^{\circ} \mathrm{C}$ & \\
\hline & & & Invierno: Máximo $4^{\circ} \mathrm{C}$ & \\
\hline
\end{tabular}

108 Reus Netto, G.; Czajkowski, J. D. 
Fuente: IRAM 11603 (INSTITUTO..., 2012).

Tabla 2 - Valores máximos de transmitancia térmica $\left(\mathrm{W} / \mathrm{m}^{2} . \mathrm{K}\right)$ para condiciones de verano

\begin{tabular}{c|c|c|c|c|c|c}
\multirow{2}{*}{$\begin{array}{c}\text { Zona } \\
\text { bioambiental }\end{array}$} & Nivel A & Nivel B & Nivel C & Nivel A & Nivel B & Nivel C \\
\cline { 2 - 7 } I y II & 0,45 & 1,10 & 1,80 & 0,18 & 0,45 & 0,72 \\
III y IV & 0,50 & 1,25 & 2,00 & 0,19 & 0,48 & 0,76 \\
\hline
\end{tabular}

Fuente: IRAM 11605 (INSTITUTO..., 1996).

Para la condición de invierno, los valores máximos admisibles están definidos en función de la temperatura exterior de diseño (TED) establecidas en la norma IRAM 11605 (INSTITUTO..., 1996), conforme se expresa en la Tabla 3.

La Temperatura Exterior de Diseño (TED) está definida en función del nivel de confort, es decir, de la temperatura interior de diseño que se considera como base, conforme se representa en la Tabla 4.

\section{Riesgo de condensación superficial e intersticial}

La norma IRAM 11625 (INSTITUTO..., 2000a) "Aislamiento térmico de edificios - Verificación de sus condiciones Higrotérmicas" establece el método para la verificación del riesgo de condensación en los paños centrales de muros exteriores, pisos y techos de edificios construidos en todas las zonas bioambientales de Argentina.

Los paños son considerados centrales hasta una distancia de $0,5 \mathrm{~m}$ de las aristas de su contorno; para puntos singulares como rincones y aristas se debe verificar en la IRAM 11630 (INSTITUTO..., 2000b).

Para cumplir con las normas citadas y evitar el riesgo de condensación, las temperaturas superficiales e intersticiales en los muros, techos y pisos no deben ser iguales o inferiores a las temperaturas de rocío correspondientes, tanto en la superficie como en todo el espesor del elemento.

El requisito de la norma exige que se debe confeccionar una planilla para cada componente de la envolvente, en donde se especifique claramente cada capa del cerramiento constructivo.

\section{Coeficiente Volumétrico de Pérdidas G cal}

La evaluación de la intensidad energética de calefacción que posee un edificio, es posible medirla a partir del Coeficiente Gcal $\left(\mathrm{W} / \mathrm{m}^{3} \mathrm{~K}\right)$ que considera las pérdidas de calor a través de los cerramientos que componen su envolvente, opacos, no opacos y en contacto con el piso, además las perdidas por renovación de aire de los locales de la vivienda calefaccionada, como lo expresa la Ecuación 1.
$\mathrm{G}=\frac{\sum \mathrm{Km} \times \mathrm{Sm}+\sum \mathrm{Kv} \times \mathrm{Sv}+\text { Per } \mathrm{xPp}}{\mathrm{V}}+0,35 \times \mathrm{n}$

$\left[\mathrm{W} / \mathrm{m}^{3 \circ} \mathrm{K}\right]$

Ec. 1

Siendo:

$\mathrm{Km}\left(\mathrm{W} / \mathrm{m}^{2} \mathrm{~K}\right)=$ transmitancia térmica de los cerramientos opacos (muros y techos);

$\mathrm{Sm}\left(\mathrm{m}^{2}\right)=$ superficies de los cerramientos opacos (muros y techos);

$\mathrm{KV}\left(\mathrm{W} / \mathrm{m}^{2 \circ} \mathrm{K}\right)=$ transmitancia térmica de los cerramientos no opacos (ventanas y puertas);

$\mathrm{Sv}\left(\mathrm{m}^{2}\right)=$ superficies dos cerramientos no opacos (ventanas y puertas);

Per $(m)=$ perímetro del piso en contacto con o aire exterior;

$\mathrm{Pp}\left(\mathrm{W} / \mathrm{m}^{\circ} \mathrm{K}\right)=$ perdida por el piso en contacto con $\mathrm{o}$ aire exterior;

$0,35\left(\mathrm{~W} / \mathrm{m}^{3 \circ} \mathrm{K}\right)=$ calor específico del aire (dato);

$\mathrm{n}=$ número de renovaciones de aire por hora (según la zona bioambiental); y

$\mathrm{V}\left(\mathrm{m}^{3}\right)=$ volumen interno a ser condicionado.

La Norma IRAM 11604 (INSTITUTO..., 2001d), titulada como "Aislamiento térmico de edificios Verificación de sus condiciones higrotérmicas. Ahorro de energía en calefacción. Coeficiente volumétrico Gcal de pérdidas de calor. Cálculo y valores límites", establece la metodología de cálculo del Coeficiente Volumétrico de Perdida de Calor (Gcal), así como también fija sus valores máximos admisibles (Tabla 5).

Está destinada a toda edificación de uso humano localizada en las zonas bioambientales III, IV, V y VI, asimismo se aplica a localidades de las demás zonas que superen los 900 grados días y no aplica a localidades que superen los 5000 grados días, en base a $18^{\circ} \mathrm{C}$.

Para cumplir con la norma el Coeficiente Gcal debe ser igual o inferior al valor máximo admisible ( $\mathrm{G}$ adm). Está calculado en función del volumen de la edificación a ser calefaccionada y sus grados días.

Los grados días son los grados acumulados en el período de un año necesarios para mantener la edificación en confort, se pueden calcular 
mediante la suma de las diferencias de temperaturas, entre la base establecida $(16,18,20$ o $22{ }^{\circ} \mathrm{C}$ ) y la media diaria, cuando la media diaria es superior a la temperatura base no se considera.

\section{Pérdidas por piso}

Corresponden a la suma de las pérdidas en contacto con el suelo contiguo a la envolvente vertical, desde el aire interior hacia el suelo o hacia al aire exterior, se calcula por metro lineal del perímetro del piso en contacto con los muros exteriores. La Tabla 6 expresa las pérdidas por piso según la zona bioambiental.

\section{Número de renovaciones $(\mathrm{n})$}

Se toma como dato que el número de renovaciones de aire en el edificio sea 2, salvo que se realice el cálculo según lo establecido en la Ecuación 2. $\mathrm{n}=\frac{\sum 0,0464 \text { li qi }\left[0,516(\mathrm{ci} \mathrm{vi})^{2}\right]^{0,67}}{\mathrm{~V}}$

Ec. 2

Siendo:

li = el largo de las juntas de contacto de cada tipo de carpinterías $(\mathrm{m})$;

qi = el caudal de infiltración de aire por unidad de longitud de la junta de cada carpintería de la envolvente del edificio $\left(\mathrm{m}^{2} / \mathrm{h}\right.$ ) (según IRAM 11.523 (INSTITUTO..., 2001));

ci $=$ el coeficiente de corrección (según Tabla B.1 IRAM 11604 (INSTITUTO..., 2001d));

vi = la velocidad del viento en la localidad considerada (m/s) (según IRAM 11.603 (INSTITUTO..., 2012)); y

$\mathrm{V}=$ el volumen de la vivienda o unidad $\left(\mathrm{m}^{3}\right)$.

Tabla 3 - Valores máximos de transmitancia térmica $(\mathrm{W} / \mathrm{m} 2 . \mathrm{K})$ para condiciones de invierno

\begin{tabular}{c|c|c|c|c|c|c}
\hline \multirow{2}{*}{ TED $\left({ }^{\mathbf{0}} \mathbf{C}\right)$} & \multicolumn{3}{|c|}{ Muros } & \multicolumn{3}{|c}{ Techos } \\
\cline { 2 - 7 } & Nivel A & Nivel B & Nivel C & Nivel A & Nivel B & Nivel C \\
$\mathbf{- 5}$ & 0,31 & 0,83 & 1,45 & 0,27 & 0,69 & 1,00 \\
$\mathbf{- 4}$ & 0,32 & 0,87 & 1,52 & 0,28 & 0,72 & 1,00 \\
$\mathbf{- 3}$ & 0,33 & 0,91 & 1,59 & 0,29 & 0,74 & 1,00 \\
$\mathbf{- 2}$ & 0,35 & 0,95 & 1,67 & 0,30 & 0,77 & 1,00 \\
$\mathbf{- 1}$ & 0,36 & 0,99 & 1,75 & 0,31 & 0,80 & 1,00 \\
$>\mathbf{0}$ & 0,38 & 1,00 & 1,85 & 0,32 & 0,83 & 1,00 \\
\hline
\end{tabular}

Fuente: IRAM 11605 (INSTITUTO..., 1996).

Tabla 4 - Temperatura interior de diseño para niveles de confort A, B y C

\begin{tabular}{cccc} 
Nivel & $\mathrm{A}\left({ }^{\circ} \mathrm{C}\right)$ & $\mathrm{B}\left({ }^{\mathrm{O}} \mathrm{C}\right)$ & $\mathrm{C}\left({ }^{\circ} \mathrm{C}\right)$ \\
\hline $\begin{array}{c}\text { Para verificar la no existencia de condensación superficial } \\
\begin{array}{c}\text { Para el confort a través de la diferencia de temperatura interior de } \\
\text { diseño y superficial de un cerramiento }\end{array}\end{array}$ & 22 & 20 & 18 \\
\hline
\end{tabular}

Fuente: IRAM 11605 (INSTITUTO..., 1996).

Tabla 5 - Valores admisibles para edificios de viviendas (Gadm)

\begin{tabular}{|c|c|c|c|c|c|c|c|c|c|}
\hline \multirow{3}{*}{$\begin{array}{c}\text { Volumen } \\
\text { Calefaccionado } \\
\left(\mathrm{m}^{3}\right)\end{array}$} & \multicolumn{9}{|c|}{ Grados dias de calefacción } \\
\hline & & & & \multicolumn{6}{|c|}{$\left({ }^{\circ} \mathrm{C}\right)$} \\
\hline & 900 & 1000 & 1100 & 1200 & 1300 & 1400 & 1500 & 2000 & 2500 \\
\hline 50 & 2,713 & 2,661 & 2,606 & 2,560 & 2,530 & 2,493 & 2,469 & 2,457 & 2,409 \\
\hline 100 & 2,213 & 2,173 & 2,133 & 2,099 & 2,077 & 2,050 & 2,032 & 2,022 & 1,986 \\
\hline 200 & 1,860 & 1,828 & 1,798 & 1,773 & 1,757 & 1,737 & 1,723 & 1,715 & 1,687 \\
\hline 300 & 1,704 & 1,676 & 1,650 & 1,629 & 1,615 & 1,598 & 1,587 & 1,579 & 1,554 \\
\hline 400 & 1,610 & 1,585 & 1,562 & 1,543 & 1,531 & 1,516 & 1,505 & 1,498 & 1,475 \\
\hline 500 & 1,547 & 1,523 & 1,502 & 1,485 & 1,473 & 1,459 & 1,449 & 1,443 & 1,421 \\
\hline 1000 & 1,389 & 1,368 & 1,352 & 1,339 & 1,330 & 1,319 & 1,311 & 1,306 & 1,287 \\
\hline 1500 & 1,319 & 1,300 & 1,286 & 1,274 & 1,266 & 1,257 & 1,250 & 1,245 & 1,228 \\
\hline 2000 & 1,277 & 1,259 & 1,246 & 1,236 & 1,228 & 1,220 & 1,213 & 1,208 & 1,193 \\
\hline 2500 & 1,249 & 1,232 & 1,219 & 1,210 & 1,203 & 1,195 & 1,188 & 1,184 & 1,169 \\
\hline 3000 & 1,228 & 1,211 & 1,199 & 1,190 & 1,184 & 1,176 & 1,170 & 1,165 & 1,151 \\
\hline 3500 & 1,211 & 1,195 & 1,184 & 1,175 & 1,169 & 1,162 & 1,156 & 1,151 & 1,137 \\
\hline 4000 & 1,198 & 1,182 & 1,171 & 1,163 & 1,157 & 1,150 & 1,144 & 1,140 & 1,126 \\
\hline 4500 & 1,187 & 1,172 & 1,161 & 1,153 & 1,147 & 1,140 & 1,135 & 1,130 & 1,117 \\
\hline 5000 & 1,178 & 1,163 & 1,152 & 1,145 & 1,139 & 1,132 & 1,127 & 1,122 & 1,109 \\
\hline 7500 & 1,147 & 1,132 & 1,123 & 1,116 & 1,110 & 1,104 & 1,099 & 1,095 & 1,082 \\
\hline 10000 & 1,128 & 1,114 & 1,105 & 1,099 & 1,093 & 1,088 & 1,083 & 1,079 & 1,067 \\
\hline
\end{tabular}

Fuente: IRAM 11604 (INSTITUTO..., 2001d). 
Tabla 6 - Pérdidas por el piso en contacto con el terreno (Pp) W/m

\begin{tabular}{|c|c|c|c|}
\hline $\begin{array}{c}\text { Zona } \\
\text { bioambiental }\end{array}$ & $\begin{array}{c}\text { Sin aislación en } \\
\text { el piso }\end{array}$ & $\begin{array}{c}\text { Aislación } \\
\text { perimetral }\end{array}$ & Aislación total \\
\hline I y II & 0,31 & 0,83 & 1,45 \\
III y IV & 0,32 & 0,87 & 1,52 \\
V y VI & 0,33 & 0,91 & 1,59 \\
\hline
\end{tabular}

Fuente: IRAM 11604 (INSTITUTO..., 2001).

\section{Calidad térmica $\mathrm{K}$ en los vidriados}

La norma IRAM 11507-1 (INSTITUTO..., 2001a) establece los requisitos básicos que deben cumplir las ventanas, con todos sus componentes, vidrios, accesorios y herrajes incluidos.

Respecto a la aislación térmica, las ventanas deben presentar un valor de transmitancia térmica total menor que $4 \mathrm{~W} / \mathrm{m}^{3} \mathrm{~K}$, este valor debe concordar con los valores de aislamiento térmico declarados por el fabricante. Como se verifica en la Tabla 7, las carpinterías pueden ser clasificadas en 5 categorías en función del desempeño térmico requerido para la zona bioambiental donde se instale la ventana. Además, K5 es para vidriados colocados en edificios de hasta $10 \mathrm{~m}$ de altura y las demás categorías para carpinterías colocadas por encima de los $10 \mathrm{~m}$ de altura.

\section{Calidad de infiltración en carpinterías}

La infiltración de aire a través de las juntas debe ser calculada según el método de la IRAM 11523 (INSTITUTO..., 2001c), y debe cumplir con los valores establecidos en la Tabla 8 (INSTITUTO..., 2001). IRAM A1 son las carpinterías colocada en edificios de hasta $10 \mathrm{~m}$ de altura e IRAM A2 de los 10 m (INSTITUTO..., 2001).

\section{Caso de estudio B: Brasil}

En Brasil existen dos normas que reglamentan el confort térmico en edificaciones, la NBR 15220 (ABNT, 2008) y NBR 15575 (ABNT, 2013). Para la elaboración de la NBR 15220 se utilizó como referencia la NBR 12538, las ASHRAE 2001 Handbook y Standart 55/92 y las ISO $7726 / 98$ (INTERNATIONAL..., 1988) y 7730/94. La NBR 15575 (ABNT, 2013) por tratarse de una norma de desempeño general de la edificación y trabajar diversos temas, cuenta con 88 normas referenciadas. Dentro del punto de vista térmico se pueden nombrar las siguientes: NBR 15215 (ABNT, 2004), 15220 (ABNT, 2008), ISO 7726, ISO 8302 (INTERNATIONAL..., 1991), UNE EN 410 (ASOCIACIÓN..., 2011), 12898, ANSI/ASHRAE 74, ASHRAE Standard 140 (AMERICABN..., 2007), ASTM C1371 (AMERICAN..., 2015a), C177 (AMERICAN...,
2013), E424-71 (AMERICAN..., 2015b), C518 y C351-92B.

La NBR 15220 (ABNT, 2008) - "Desempeño Térmico de Edificaciones", establecida sin carácter normativo, se destina a mejorar la calidad de las viviendas unifamiliares de interés social hasta 3 pisos. Se enfoca en el confort térmico y propone un conjunto de recomendaciones y estrategias de diseño que deben ser aplicadas a la construcción en función de la zona bioclimática en que se encuentra.

\section{Clima}

El territorio brasileño fue dividido en 8 zonas, conforme se ilustra en la Figura 2, según las características bioclimáticas mencionadas en la Tabla 9.

\section{Requisitos y criterios mínimos}

A nivel normativo, la NBR 15575 (ABNT, 2013) establece un nivel mínimo de desempeño que debe ser atendido por todas las nuevas edificaciones de tipo residencial con un número indeterminado de niveles.

A diferencia de la NBR 15220 (ABNT, 2008), la NBRF 15575 (ABNT, 2013) busca alcanzar un nivel mínimo de desempeño para cada sistema de la obra: estructural, pisos, cerramientos verticales, cubiertas e hidrosanitario, siendo que para cada sistema establece requisitos y criterios para garantizar la habitabilidad (estanquidad, desempeño térmico, acústico y lumínico, salud, higiene y calidad del aire, funcionamiento y accesibilidad, confort táctil y antropodinamico), la seguridad (estructural, contra incendio y durante el uso y operación) y la sustentabilidad (durabilidad, mantenimiento y adecuación ambiental).

Para algunos criterios la norma indica otros dos niveles de desempeño opcional, Intermedio (I) y Superior (S).

Para cumplir con la NBR 15575 (ABNT, 2013) los criterios generales deben ser obligatoriamente atendidos por todos los sistemas y elementos de la edificación. Además de todos los requisitos generales, la norma define el tiempo mínimo en que cada sistema debe ser atendido, presumiendo 
el correcto mantenimiento, para ello solicita que los proyectistas indiquen y especifiquen la Vida Útil de Proyecto (VUP) y suministren un Manual de Uso y Operación que la constructora debe entregar al usuario para que realice el mantenimiento necesario y garantice la vida útil descrita en la normativa.

La evaluación de los criterios de desempeño térmico puede ser realizada de dos formas, por el modo simplificado en donde el proyectista informa a través de cálculos que la envolvente cumple con los criterios de transmitancia térmica y capacidad térmica establecidos por cada cerramiento, o a través del simulado con EnergyPlus, cuando los valores obtenidos en el primer momento sean insatisfactorios.

\section{Metodología simplificada}

Las edificaciones deben cumplir con los valores mínimos de transmitancia térmica, capacidad térmica y absorción a la radiación, indicados en la Tabla 10.

Conforme establecido en la NBR 15220-3 (ABNT, 2008)el factor de ventilación (FV) varía en función de la dimensión de las aperturas de ventilación presente en los aleros, conforme la Equación 3, siendo que para cubiertas sin cielorraso o áticos no ventilados $\mathrm{FV}=1$.

$\mathrm{FV}=1,17-1,07 \cdot \mathrm{h}^{-1,04}$

Ec. 3

Siendo $\mathrm{h}=$ la altura de la abertura en los dos aleros (cm).

Tabla 7 - Categoría de Aislación Térmica

\begin{tabular}{|c|c|}
\hline $\begin{array}{l}\text { Categoría de } \\
\text { Aislación }\end{array}$ & $\begin{array}{c}\text { Transmitancia térmica } \\
\qquad K\left(e n ~ W / m^{2} K\right)\end{array}$ \\
\hline $\mathrm{K}_{1}$ & $\mathrm{~K}<1,0$ \\
\hline $\mathrm{K}_{2}$ & $1,0 \leq \mathrm{K} \leq 1,5$ \\
\hline $\mathrm{K}_{3}$ & $1,5 \leq \mathrm{K} \leq 2,0$ \\
\hline $\mathrm{K}_{4}$ & $2,0 \leq \mathrm{K} \leq 3,0$ \\
\hline $\mathrm{K}_{5}$ & $3,0 \leq \mathrm{K} \leq 4,0$ \\
\hline No clasificable & $\mathrm{K}>4,0$ \\
\hline
\end{tabular}

Fuente: IRAM 11507 (INSTITUTO..., 2001b).

Tabla 8 - Categoría de Aislación Térmica

\begin{tabular}{c|c|c}
\hline $\begin{array}{c}\text { Clasificación según la } \\
\text { infiltración }\end{array}$ & $\begin{array}{c}\text { Caudal de aire por metro } \\
\text { de junta }\left(\mathbf{m}^{\mathbf{3}} / \mathbf{h} \cdot \mathbf{m}\right)\end{array}$ & Designación \\
IRAM A1 & Mayor que 4,01 hasta 6,00 & Normal \\
IRAM A2 & Mayor que 2,01 hasta 4,00 & Mejorada \\
IRAM A3 & Hasta 2,00 & Reforzada \\
\hline
\end{tabular}

Fuente: IRAM 11507 (INSTITUTO..., 2001b).

Figura 2 - Mapa bioambiental Brasileño

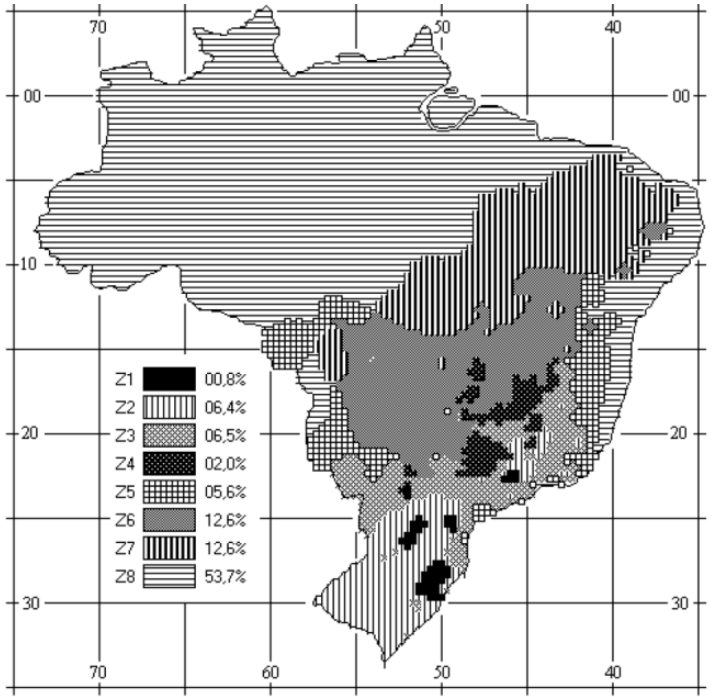

112 Reus Netto, G.; Czajkowski, J. D. 
Fuente: NBR 15220 (ABNT, 2008).

Tabla 9 - Características de las zonas bioclimáticas de Brasil

\begin{tabular}{|c|c|c|c|}
\hline Clasificación & Zona & $\begin{array}{l}\text { Temperatura media } \\
\text { diária * }\end{array}$ & $\begin{array}{c}\text { Amplitud } \\
\text { media anual ** }\end{array}$ \\
\hline I - Más fría & Pequeñas localidades en el sur & Entre $0,7^{\circ} \mathrm{C}$ y $31,4^{\circ} \mathrm{C}$ & $9,0^{\circ} \mathrm{C}$ \\
\hline II & Sur del país & Medias de $18^{\circ} \mathrm{C}^{* *}$ & $11,0^{\circ} \mathrm{C}$ \\
\hline III & Predominancia en el centro sur & Entre $6,0^{\circ} \mathrm{C}$ y $35,9^{\circ} \mathrm{C}$ & $8,0^{\circ} \mathrm{C}$ \\
\hline IV & Pequeñas localidades en el centro oeste & Entre $10,0^{\circ} \mathrm{C}$ y $31,2^{\circ} \mathrm{C}$ & $11,0^{\circ} \mathrm{C}$ \\
\hline $\mathbf{V}$ & $\begin{array}{l}\text { Franjas de extensión norte-sur en el } \\
\text { centro oeste y centro leste }\end{array}$ & Medias de $22^{\circ} \mathrm{C}^{* *}$ & $8,0^{\circ} \mathrm{C}$ \\
\hline VI & Predominancia en el centro & Entre $9,6^{\circ} \mathrm{C}$ y $34,6^{\circ} \mathrm{C}$ & $11,5^{\circ} \mathrm{C}$ \\
\hline VII & Predominancia en el nordeste del país & Entre $11,4^{\circ} \mathrm{C}$ y $37,8^{\circ} \mathrm{C}$ & $11,5^{\circ} \mathrm{C}$ \\
\hline $\begin{array}{c}\text { VIII - } \\
\text { Caliente y } \\
\text { húmedo }\end{array}$ & $\begin{array}{l}\text { Predominancia en la zona Amazónica } \\
\text { con una extensión por la costa nordeste }\end{array}$ & Entre $11,9^{\circ} \mathrm{C}$ y $37,9^{\circ} \mathrm{C}$ & $8,5^{\circ} \mathrm{C}$ \\
\hline
\end{tabular}

Fuente: NBR 15220 (ABNT, 2008).

Nota: Leyenda:

*Las temperaturas medias fueron tomadas como promedio de las localidades disponibles en la NBR 15.220.

** Por no disponer de datos en la NBR 15.220 se tomaron las temperaturas medias promedio y las amplitudes medias anuales establecidos en la Classificação de climas do Brasil - Versão 2 - Maurício Roriz - ANTAC:

Associação Nacional de Tecnologia do Ambiente Construído. Grupo de Trabalho sobre Conforto Ambiental e Eficiência Energética de Edificações. São Carlos, SP. Novembro de 2013.

Tabla 10 - Valores de Transmitancia Térmica (W/m3.K), Capacidad térmica (kJ/m2.K) y Absorción a la radiación para muros y techos

\begin{tabular}{|c|c|c|c|c|c|c|c|}
\hline \multirow{3}{*}{$\begin{array}{c}\text { Zona } \\
\text { bioambiental }\end{array}$} & \multicolumn{3}{|c|}{ Muros } & \multicolumn{4}{|c|}{ Techos } \\
\hline & \multirow{2}{*}{$\begin{array}{c}\text { Transmitancia } \\
\text { Térmica }\end{array}$} & \multirow{2}{*}{$\begin{array}{l}\text { Capacidad } \\
\text { Térmica }\end{array}$} & \multirow{2}{*}{$\begin{array}{c}\text { Absorción } \\
\text { a la } \\
\text { radiación }\end{array}$} & \multicolumn{3}{|c|}{ Transmitancia Térmica } & \multirow{2}{*}{$\begin{array}{c}\text { Absorció } \\
\mathrm{n} \text { a la } \\
\text { radiación }\end{array}$} \\
\hline & & & & M & I & $S$ & \\
\hline 1 y 2 & $\leq 2,5$ & $\geq 130$ & - & $\leq 2,3$ & $\leq 1,5$ & $\leq 1,0$ & - \\
\hline \multirow{2}{*}{$3,4,5$ y 6} & $\leq 3,7$ & $\geq 130$ & $\alpha^{a} \leq 0,6$ & $\leq 2,3$ & $\leq 1,5$ & $\leq 1,0$ & $\alpha \leq 0,6$ \\
\hline & $\leq 2,5$ & $\geq 130$ & $\alpha^{a}>0,6$ & $\leq 1,5$ & $\leq 1,0$ & $\leq 0,5$ & $\alpha>0,6$ \\
\hline \multirow{2}{*}{7 y $8^{*}$} & $\leq 3,7$ & $\geq 130$ & $\alpha^{a} \leq 0,6$ & $\leq 2,3 \mathrm{FV}$ & $\leq 1,5 \mathrm{FV}$ & $\leq 1,0 \mathrm{FV}$ & $\alpha \leq 0,4$ \\
\hline & $\leq 2,5$ & $\geq 130$ & $\alpha^{a}>0,6$ & $\leq 1,5 \mathrm{FV}$ & $\leq 1,0 \mathrm{FV}$ & $\leq 0,5 \mathrm{FV}$ & $\alpha>0,4$ \\
\hline
\end{tabular}

Fuente: NRB 15575 (ABNT, 2013).

Nota: *Sin requisito para Capacidad térmica.

\section{Metodología simulación energética}

Para analizar el desempeño térmico global de la edificación, la NBR 15575 (ABNT, 2013) exige que los profesionales utilicen el EnergyPlus u otro programa validado por ASHRAE Standard 140 (AMERICAN..., 2007) que permita la determinación del comportamiento térmico edilicio sobre condiciones dinámicas de exposición al clima.

Para la carga de datos en el software, deben utilizarse las tablas presentes en el Anexo A de la NBR 15575-1 (ABNT, 2013), que brindan información sobre la localización geográfica de algunas ciudades brasileras y datos climáticos relacionados a los días típicos de verano e invierno. Para las ciudades no consideradas en la tabla, se recomienda utilizar los datos de una ciudad próxima, ubicada en la misma zona bioclimática, con altitud y características climáticas semejantes.

\section{Temperaturas de diseño}

Los días típicos de invierno y verano, se definen a partir de la temperatura del aire, de la humedad relativa, de la velocidad del viento y la radiación incidente en el día más frio y para el más caluroso del año.

\section{Requisito de desempeño en verano}

Como desempeño térmico mínimo, los ambientes de permanencia prolongada, tales como el estar y dormitorios, sin presencia de fuentes internas de calor (ocupantes, lámparas y otros equipos en general), deben presentar un valor diario máximo de temperatura igual o inferior al valor máximo 
diario de la temperatura del aire exterior, conforme indicado en la Tabla 11.

\section{Requisito de desempeño en invierno}

Para cumplir con los requisitos de invierno, los valores mínimos diarios de la temperatura del aire en los ambientes de larga permanencia de edificaciones localizadas en las zonas de 1 a 5 , deben ser superiores o igual a la temperatura mínima media externa sumado $3^{\circ} \mathrm{C}$. Las edificaciones ubicadas en las demás zonas no requieren cumplir con este requisito.

\section{Renovaciones de aire}

La norma aclara detalles importantes para la correcta evaluación de la simulación energética y establece que para la ventilación y la protección de aberturas puede considerarse valores estándares. Se establece una tasa de $1 \mathrm{ren} / \mathrm{h}$ cuando no se verifica una protección solar sobre la venta, contra la entrada de radiación.

\section{Pisos}

La norma no define requisitos específicos para el desempeño térmico de los sistemas de pisos. Los requisitos de análisis global de desempeño térmico de las edificaciones son considerados en la simulación computacional.

\section{Aberturas y ventilación}

Los ambientes de larga permanencia, tales como el estar, la cocina y los dormitorios, deben tener una superficie de ventilación según lo indicado en la Tabla 12.

\section{Discusión}

De la investigación del material referente a las normas que tratan del desempeño térmico edilicio en Argentina y Brasil, se pudo determinar sus características y con eso elaborar la Tabla 13, en la cual se señala las principales coincidencias y divergencias.

La preocupación en regular la construcción para garantizar el confort térmico en Argentina es anterior a Brasil. Argentina en 1970 ya presentaba una normativa con recomendaciones generales de diseño, su respectivo mapa bioambiental y los valores máximos admisibles para transmitancia térmica, mientras en Brasil esas directivas aparecieron en 2005.

Para el cumplimiento de ambas normativas, se verifica como método de cálculo un sistema simplificado, donde el profesional debe presentar junto al proyecto los cálculos que corroboren cumplir con los valores establecidos.

Se observa desde el punto de vista legal que la NBR 15575 (ABNT, 2013) tiene carácter obligatorio y las normas IRAM informativo, necesitando de leyes, decretos y códigos de edificaciones para ser obligatorias. En Argentina una ley de la Provincia de Buenos Aires las constituyo como obligatorias en 2003, en 2012 una ley en la ciudad de Buenos Aires y en 2010 un código de edificación en la ciudad de Rosario, sin embargo se presume que no hay fiscalización formalizada por parte de las autoridades y para ambas normativas es difícil de lograr su efectiva implementación, no se cumplen o lo hacen parcialmente.

Tabla 11 - Criterio para evaluación de desempeño térmico para verano e invierno

\begin{tabular}{cc|c|c|c} 
Nivel de & \multicolumn{2}{c}{ Condición de verano } & \multicolumn{2}{c}{ Condición de invierno } \\
desempeño & Zona 1 a 7 & Zona 8 & Zona 1 a 5 & Zona 6,7 y 8 \\
\hline Mínimo & $\mathrm{Ti}, \max \leq \mathrm{Te}, \max *$ & $\mathrm{Ti}, \max \leq \mathrm{Te}, \max$ & $\mathrm{Ti}, \min \geq \mathrm{Te}, \min +3{ }^{\circ} \mathrm{C}$ & - \\
Intermedio & $\mathrm{Ti}, \max \leq \mathrm{Te}, \max -2^{\circ} \mathrm{C}$ & $\mathrm{Ti}, \max \leq \mathrm{Te}, \max -1{ }^{\circ} \mathrm{C}$ & $\mathrm{Ti}, \min \geq \mathrm{Te}, \min +5{ }^{\circ} \mathrm{C}$ & - \\
Superior & $\mathrm{Ti}, \max \leq \mathrm{Te}, \max -4^{\circ} \mathrm{C}$ & $\mathrm{Ti}, \max \leq \mathrm{Te}, \max -2{ }^{\circ} \mathrm{C}$ & $\mathrm{Ti}, \min \geq \mathrm{Te}, \min +7{ }^{\circ} \mathrm{C}$ & - \\
\hline
\end{tabular}

Fuente: NRB 15575 (ABNT, 2013).

Nota: Leyenda:

*Ti,máx/mím es el valor máximo/mínimo diario de la temperatura del aire en la edificación $\left({ }^{\circ} \mathrm{C}\right)$; y

**Te,máx/mím es el valor máximo/mínimo diario de la temperatura exterior a la edificación $\left({ }^{\circ} \mathrm{C}\right)$.

Tabla 12 - Superficie mínima de ventilación para dormitorios y estar

\begin{tabular}{c|c|c}
$\begin{array}{c}\text { Nivel de } \\
\text { desempeño }\end{array}$ & $\begin{array}{c}\text { Zona } 1 \text { a } 7 \text { aperturas } \\
\text { medianas }\end{array}$ & Condición de verano \\
\hline M & $\begin{array}{c}\mathrm{A} \geq 7 \% \text { de la superficie } \\
\text { del piso }\end{array}$ & $\begin{array}{c}\mathrm{A} \geq 12 \% \text { grandes aperturas la superficie del piso para región Norte } \\
\mathrm{A} \geq 8 \% \text { de la superficie del piso para Nordeste y Sudeste }\end{array}$ \\
\hline
\end{tabular}

Fuente: NRB 15575 (ABNT, 2013).

114 Reus Netto, G.; Czajkowski, J. D. 
Tabla 13 - Grafico síntesis comparativo entre normativa de desempeño térmico edilicio de Argentina y Brasil

\begin{tabular}{|c|c|c|c|c|c|c|c|}
\hline \multirow{2}{*}{ Características } & \multicolumn{5}{|c|}{ ARGENTINA } & \multicolumn{2}{|c|}{ BRASIL } \\
\hline & $\begin{array}{c}11601 \\
\text { (INSTITUTO.. } \\
., 2002) \\
\end{array}$ & $\begin{array}{c}11603 \\
\text { (INSTITUTO... } \\
., 2012) \\
\end{array}$ & $\begin{array}{c}11604 \\
\text { (INSTITUTO..., } \\
\text { 2001d) }\end{array}$ & $\begin{array}{c}11605 \\
(\text { INSTITUTO... } \\
., 1996) \\
\end{array}$ & $\begin{array}{c}11625-11630 \\
\text { (INSTITUTO..., } \\
\text { 2000a, 2000b) }\end{array}$ & $\begin{array}{c}15220 \\
(\mathrm{ABNT}, 2008)\end{array}$ & $\begin{array}{c}15575 \\
(\mathrm{ABNT}, 2013)\end{array}$ \\
\hline \multirow{2}{*}{$\begin{array}{c}\text { Año* } \\
\text { Carácter }\end{array}$} & 2002 & 2012 & 2001 & 1996 & 2000 & 2005 & \multirow{2}{*}{$\begin{array}{c}2013 \\
\text { Obligatorio }\end{array}$} \\
\hline & \multicolumn{5}{|c|}{ Informativo / Obligatorio para vivienda de interés social } & Informativo & \\
\hline Revisión de & $\begin{array}{l}\text { IRAM } 11601 \\
\quad(1996)\end{array}$ & $\begin{array}{l}\text { IRAM } \\
11603 \\
(1996)\end{array}$ & $\begin{array}{l}\text { IRAM } 11604 \\
\qquad(1990)\end{array}$ & $\begin{array}{l}\text { IRAM } \\
11605 \\
(1980) \\
\end{array}$ & $\begin{array}{l}\text { IRAM 11625 } \\
\text { y 11.630; } \\
(1991)\end{array}$ & $\begin{array}{l}\text { NBR, ASHRAE } \\
\text { y ISO. }\end{array}$ & $\begin{array}{l}\text { ABNT, UNE, } \\
\text { ISO, ASTM y } \\
\text { Eurocode }\end{array}$ \\
\hline $\begin{array}{l}\text { Responsable } \\
\text { por la } \\
\text { elaboración }\end{array}$ & \multicolumn{5}{|c|}{ IRAM, Comité de normas y Subcomité de aislamiento térmico de edificios } & $\begin{array}{l}\text { ABNT, } \\
\text { Comisiones de } \\
\text { estudios }\end{array}$ & $\begin{array}{c}\text { ABNT, Secovi, } \\
\text { Sinduscon, } \\
\text { IPT, USP, } \\
\text { CAIXA. } \\
\text { Universidades } \\
\text { y profesionales }\end{array}$ \\
\hline Objetivo & \multicolumn{5}{|c|}{$\begin{array}{c}\text { Definir las condiciones de acondicionamiento térmico y garantizar un correcto } \\
\text { aislamiento térmico para las edificaciones }\end{array}$} & $\begin{array}{c}\text { Mejorar el } \\
\text { confort térmico } \\
\text { de las viviendas }\end{array}$ & $\begin{array}{c}\text { Alcanzar un } \\
\text { nivel mínimo } \\
\text { de desempeño } \\
\text { para cada } \\
\text { sistema }\end{array}$ \\
\hline  & $\begin{array}{l}\text { Métodos de } \\
\text { cálculo }\end{array}$ & $\begin{array}{c}\text { Mapa } \\
\text { Bioambient } \\
\text { al }\end{array}$ & $\begin{array}{c}\text { Valores } \\
\text { máximos } \\
\text { admisibles } \\
\text { para el } \\
\text { Coef.G de } \\
\text { calefacción y } \\
\text { método de } \\
\text { cálculo }\end{array}$ & $\begin{array}{l}\text { Valores } \\
\text { máximos } \\
\text { de } \\
\text { transmitanc } \\
\text { ia térmica } \\
\text { para muros } \\
\text { y techos }\end{array}$ & $\begin{array}{c}\text { Método para } \\
\text { la } \\
\text { verificación } \\
\text { del riesgo de } \\
\text { condensación } \\
\text { superficial e } \\
\text { intersticial }\end{array}$ & $\begin{array}{l}\text { Métodos de } \\
\text { cálculo }\end{array}$ & $\begin{array}{c}\text { Valores límites } \\
\text { de Transm. } \\
\text { térmica para } \\
\text { muros y techos }\end{array}$ \\
\hline \multirow{3}{*}{ 总 } & - & - & $\begin{array}{l}\text { Comprobar el } \\
\text { valor de Gcal }\end{array}$ & $\begin{array}{l}\text { Cumplir } \\
\text { con los } \\
\text { valores } \\
\text { máximos } \\
\text { de K } \\
\text { admisible }\end{array}$ & $\begin{array}{l}\text { Verificar con } \\
\text { el riesgo de } \\
\text { condensación }\end{array}$ & - & $\begin{array}{c}\text { Atender } \\
\text { necesariamente } \\
\text { a los requisitos } \\
\text { generales }\end{array}$ \\
\hline & - & - & - & - & - & - & $\begin{array}{l}\text { Cumplir con } \\
\text { los valores } \\
\text { mínimos de } \\
\text { transmitancia } \\
\text { térmica para } \\
\text { muros y techos }\end{array}$ \\
\hline & - & - & - & - & - & - & $\begin{array}{l}\text { Alcanzar los } \\
\text { valores } \\
\text { mínimos de } \\
\text { Cap. Térmica } \\
\text { para muros** }\end{array}$ \\
\hline \multirow{2}{*}{\begin{tabular}{c|} 
Métodos de \\
cálculo
\end{tabular}} & Simplificado & Simplificado & Simplificado & Simplificado & Simplificado & - & Simplificado \\
\hline & - & - & - & - & - & - & Simulado \\
\hline
\end{tabular}

Nota: Leyenda:

*Última actualización - Revisión; y

${ }^{* *}$ Cuando empleado al método de simulación Faixa de Temperatura. 
Según la opinión de algunos profesionales en Brasil (SILVA et al., 2011) una de las problemáticas para la aplicación de las normas es la falta de profesionales calificados con el conocimiento para el desarrollo de todos los requisitos del proyecto, la mano de obra, y el mayor tiempo necesario para el desarrollo del proyecto completo. La mayoría de los entrevistados opinan que es necesario un mayor tiempo de adaptación previo a ser exigida de manera intensiva, expresan la necesidad que haya un profesional responsable solamente para la implementación de la normativa en los estudios de arquitectura y constructoras.

En los requisitos exigidos por ambas normas, se observa que las normas IRAM trabajan la calefacción de manera activa, establece valores máximos admisibles para el coeficiente $G$ de calefacción y determinan el valor máximo para la carga térmica anual donde un edificio debe cumplir con determinados valores de transmitancia térmica en sus cerramientos, conjuntamente con un control de las perdidas por infiltración de manera que se garantice que el valor del Coeficiente Gcal no sea superior al establecido en la norma, en función del volumen de la edificación y su zona bioambiental.

Según las características climáticas del territorio argentino, sumado al sistema constructivo utilizado convencionalmente, las normas IRAM establecen los requisitos para verificar el riesgo de condensación, es decir, que las temperaturas superficiales e intersticiales en los muros, techos y pisos NO deben ser iguales o inferiores a las temperaturas de rocío correspondientes, tanto en la superficie como en todo el espesor del elemento, evitando de esa forma que al pasar el aire del interior hacia el exterior, condense a dentro del elemento constructivo generando patologías como la aparición de hongos, humedad.

Se estima que el coeficiente Gcal de calefacción, la carga anual térmica, así como los riesgos de condensación no son tratados en las normas brasileñas ya que las características climáticas de invierno en Brasil no son tan rigurosas como en Argentina. Una vez que se verifica la aparición de patologías en diversas edificaciones en territorio brasilero relacionadas a infiltración y condensación en las superficies, se plantea la importancia de implementar a la NBR 15575 (ABNT, 2013) un sistema de verificación de riesgo de condensación y quizás el cálculo de las pérdidas de calor para las zonas más frías.

Desde el punto de vista de la transmitancia térmica y con el fin de establecer una comparación entre los niveles exigidos en las normas, se organizó las Tablas 14 y 15 que describen las temperaturas medias de las principales localidades de cada zona bioclimática de Argentina y Brasil y su relación con los valores de Transmitancia Térmica K establecidos por cada normativa.

En el análisis de las Figuras 3 y 4 que grafica los valores de Transmitancia Térmica $\mathrm{K}$ exigidos por las normas en relación a las temperaturas medias de cada localidad, se establece un rango en donde coinciden las temperaturas medias de algunas ciudades de Argentina y Brasil y se verifica que a la vez, los valores máximos de transmitancia térmica son bastante diferentes. Se infiere que la NBR es un $108 \%$ más permisiva que las IRAM en verano y $177 \%$ y $257 \%$ en invierno, para techos y muros respectivamente. Este factor puede indicar porqué aunque existan viviendas que cumplan con los valores determinados en la norma, no es posible obtener confort en los ambientes internos (GRIGOLETTI; LINCK, 2014; GONÇALVES; DUARTE, 2006). 
Tabla 14 - Relación de localidades y sus temperaturas mínimas, medias y máximas para invierno y verano de Argentina

\begin{tabular}{|c|c|c|c|c|c|c|c|c|c|}
\hline \multicolumn{10}{|c|}{ ARGENTINA } \\
\hline \multirow{2}{*}{\multicolumn{2}{|c|}{ Zona }} & \multirow{2}{*}{$\begin{array}{c}\text { Localidad (*Capital de la } \\
\text { província) }\end{array}$} & \multirow{2}{*}{ Provincia } & \multicolumn{3}{|c|}{ INVIERNO * } & \multicolumn{3}{|c|}{ VERANO* } \\
\hline & & & & TMIN & TMED & TMAX & TMIN & TMED & TMAX \\
\hline I & $\mathrm{a}$ & La Rioja* & La Rioja & 6,70 & 13,89 & 21,10 & 20,00 & 26,74 & 33,30 \\
\hline I & $\mathrm{a}$ & Santiago del Estero* & Santiago del Estero & 7,40 & 14,77 & 22,20 & 19,60 & 26,13 & 32,60 \\
\hline I & $\mathrm{b}$ & Resistencia* & Chaco & 10,90 & 16,76 & 22,50 & 20,10 & 26,10 & 32,10 \\
\hline I & $\mathrm{b}$ & Corrientes* & Corrientes & 11,70 & 16,93 & 22,30 & 20,40 & 26,11 & 31,90 \\
\hline I & $\mathrm{b}$ & Formosa* & Formosa & 13,10 & 18,32 & 23,60 & 21,50 & 27,15 & 32,80 \\
\hline I & $\mathrm{b}$ & Posadas* & Misiones & 12,70 & 17,86 & 23,00 & 20,90 & 26,57 & 32,20 \\
\hline II & $\mathrm{a}$ & $\begin{array}{l}\text { San F. del Valle de } \\
\text { Catamarca* }\end{array}$ & Catamarca & 6,80 & 14,35 & 21,90 & 20,50 & 26,78 & 33,10 \\
\hline II & $\mathrm{b}$ & Paraná* & Entre Ríos & 8,50 & 13,39 & 18,30 & 18,20 & 23,86 & 29,60 \\
\hline II & $\mathrm{b}$ & Santa Fe - Sauce Viejo & Santa Fe & 8,60 & 13,84 & 19,00 & 19,00 & 24,60 & 30,20 \\
\hline II & $\mathrm{b}$ & San Miguel de Tucumán* & Tucumán & 8,70 & 14,62 & 20,50 & 19,30 & 24,47 & 29,30 \\
\hline III & $\mathrm{a}$ & Junín & Buenos Aires & 5,10 & 10,93 & 16,80 & 15,40 & 21,93 & 28,40 \\
\hline III & $\mathrm{a}$ & Córdoba* & Córdoba & 5,80 & 12,47 & 19,10 & 16,50 & 22,35 & 28,30 \\
\hline III & $\mathrm{a}$ & Santa Rosa* & La Pampa & 3,50 & 9,77 & 16,00 & 15,00 & 22,21 & 29,40 \\
\hline III & $\mathrm{a}$ & Salta* & Salta & 4,90 & 12,74 & 20,60 & 15,80 & 21,26 & 26,70 \\
\hline III & $\mathrm{a}$ & San Juan* & San Juan & 2,70 & 10,61 & 18,50 & 18,00 & 25,56 & 33,10 \\
\hline III & $\mathrm{a}$ & San Luis* & San Luis & 5,40 & 11,77 & 18,20 & 16,70 & 23,23 & 29,70 \\
\hline III & $\mathrm{a}$ & Rosario & Santa Fe & 6,50 & 12,20 & 17,90 & 17,20 & 23,27 & 29,30 \\
\hline III & $\mathrm{b}$ & La Plata* & Buenos Aires & 6,50 & 11,08 & 15,70 & 16,40 & 21,89 & 27,40 \\
\hline III & $\mathrm{b}$ & San Salvador de Jujuy* & Jujuy & 7,70 & 14,45 & 21,20 & 17,70 & 23,40 & 29,10 \\
\hline IV & $\mathrm{a}$ & Mendoza* & Mendoza & 3,90 & 10,47 & 16,90 & 17,30 & 24,06 & 30,70 \\
\hline IV & $\mathrm{a}$ & Neuquén* & Neuquén & 1,70 & 8,11 & 14,50 & 13,90 & 21,95 & 29,90 \\
\hline IV & $\mathrm{c}$ & Bahía Blanca & Buenos Aires & 4,00 & 9,60 & 15,30 & 14,80 & 22,00 & 29,20 \\
\hline IV & $\mathrm{c}$ & Mar del Plata & Buenos Aires & 4,40 & 9,38 & 14,40 & 13,60 & 19,43 & 25,20 \\
\hline IV & $\mathrm{c}$ & Rawson - Trelew & Chubut & 1,60 & 7,64 & 13,80 & 12,20 & 19,87 & 27,50 \\
\hline IV & $\mathrm{c}$ & Viedma* & Río Negro & 2,70 & 8,45 & 14,30 & 13,40 & 20,54 & 27,80 \\
\hline VI & & Río Gallegos* & Santa Cruz & $-1,20$ & 2,59 & 6,40 & 6,70 & 12,81 & 18,90 \\
\hline VI & & Ushuaia* & Tierra del Fuego & $-0,40$ & 2,54 & 5,40 & 5,00 & 9,32 & 13,50 \\
\hline
\end{tabular}

Fuente: Norma IRAM 11603 (INSTITUTO..., 2012).

Nota: *Temperaturas media, máxima y mínima medias promedio de los meses de invierno y verano $\left({ }^{\circ} \mathrm{C}\right)$ IRAM 11603 (INSTITUTO..., 2012). 
Tabla 15 - Relación de localidades y sus temperaturas para invierno y verano de Brasil

\begin{tabular}{|c|c|c|c|c|c|c|c|c|}
\hline \multicolumn{9}{|c|}{ BRASIL } \\
\hline \multirow{2}{*}{ Zona } & \multirow{2}{*}{ Capital } & \multirow{2}{*}{ Provincia } & \multicolumn{3}{|c|}{ INVIERNO } & \multicolumn{3}{|c|}{ VERANO } \\
\hline & & & TMIN & TMED & TMAX & TMIN & TMED & TMAX \\
\hline 1 & Curitiba & Paraná & 8,10 & 12,90 & 19,40 & 16,30 & 20,60 & 26,70 \\
\hline 3 & Belo Horizonte & Minas Gerais & 13,10 & 18,00 & 24,6 & 19,00 & 23,00 & 28,8 \\
\hline 3 & Porto Alegre & Rio Grande do Sul & 10,70 & 14,70 & 19,40 & 20,80 & 24,60 & 30,10 \\
\hline 3 & Florianópolis & Santa Catarina & 13,30 & 16,50 & 20,40 & 21,80 & 24,60 & 28,40 \\
\hline 3 & São Paulo & São Paulo & 11,70 & 15,80 & 21,80 & 18,80 & 22,40 & 28,00 \\
\hline 4 & Brasília & Distrito Federal & 12,90 & 18,30 & 25,10 & 17,40 & 21,20 & 26,90 \\
\hline 6 & Goiânia & Goiás & 13,20 & 20,90 & 28,90 & 19,70 & 23,80 & 29,40 \\
\hline 6 & Campo Grande & Mato Grosso do Sul & 14,50 & 20,30 & 28,10 & 20,40 & 24,60 & 30,10 \\
\hline 7 & Cuiabá & Mato Grosso & 17,00 & 22,80 & 31,80 & 23,20 & 26,70 & 32,60 \\
\hline 7 & Palmas & Tocantins & 15,40 & 22,90 & 32,00 & 20,80 & 25,30 & 30,80 \\
\hline 8 & Teresina & Piauí & 20,40 & 26,20 & 33,30 & 22,50 & 26,70 & 32,20 \\
\hline 8 & Rio Branco & Acre & 16,50 & 23,20 & 31,30 & 21,30 & 25,30 & 30,90 \\
\hline 8 & Maceió & Alagoas & 20,50 & 23,60 & 27,00 & 22,60 & 26,50 & 30,40 \\
\hline 8 & Macapá & Amapá & 22,90 & 26,10 & 30,60 & 23,10 & 25,70 & 29,20 \\
\hline 8 & Manaus & Amazonas & 22,70 & 26,50 & 31,30 & 23,10 & 26,10 & 30,50 \\
\hline 8 & Salvador & Bahia & 21,40 & 23,70 & 26,20 & 23,60 & 26,40 & 29,90 \\
\hline 8 & Fortaleza & Ceará & 22,40 & 25,60 & 29,50 & 24,40 & 27,10 & 30,50 \\
\hline 8 & Vitória & Espírito Santo & 18,80 & 21,70 & 25,90 & 23,10 & 26,30 & 30,90 \\
\hline 8 & São Luís & Maranhão & 22,60 & 25,90 & 30,40 & 23,50 & 26,10 & 30,00 \\
\hline 8 & Belém & Pará & 21,70 & 25,70 & 31,70 & 22,20 & 25,40 & 30,50 \\
\hline 8 & João Pessoa & Paraíba & 20,90 & 24,20 & 27,50 & 23,50 & 27,20 & 30,50 \\
\hline 8 & Recife & Pernambuco & 21,10 & 23,90 & 27,30 & 22,60 & 26,50 & 30,20 \\
\hline 8 & Rio de Janeiro & Rio de Janeiro & 18,40 & 21,30 & 25,00 & 23,50 & 26,60 & 30,20 \\
\hline 8 & Natal & Rio Grande do Norte & 20,80 & 24,30 & 27,70 & 24,00 & 27,00 & 30,20 \\
\hline 8 & Porto Velho & Rondônia & 18,30 & 24,60 & 32,70 & 21,50 & 25,50 & 31,50 \\
\hline 8 & Boa Vista & Roraima & 22,40 & 25,90 & 30,30 & 23,30 & 28,00 & 33,40 \\
\hline 8 & Aracaju & Sergipe & 21,60 & 24,40 & 27,00 & 24,30 & 27,00 & 29,60 \\
\hline
\end{tabular}

Fuente: IRAM 11603 (INSTITUTO..., 2012), NBR 15575 (ABNT, 2013) y Instituto Nacional de Metereologia do Brasil (INMET) $)^{5}$.

Figura 3 - Relación entre las temperaturas medias de verano de las localidades de Argentina y Brasil, en función de los valores de Kadm para muros y techos

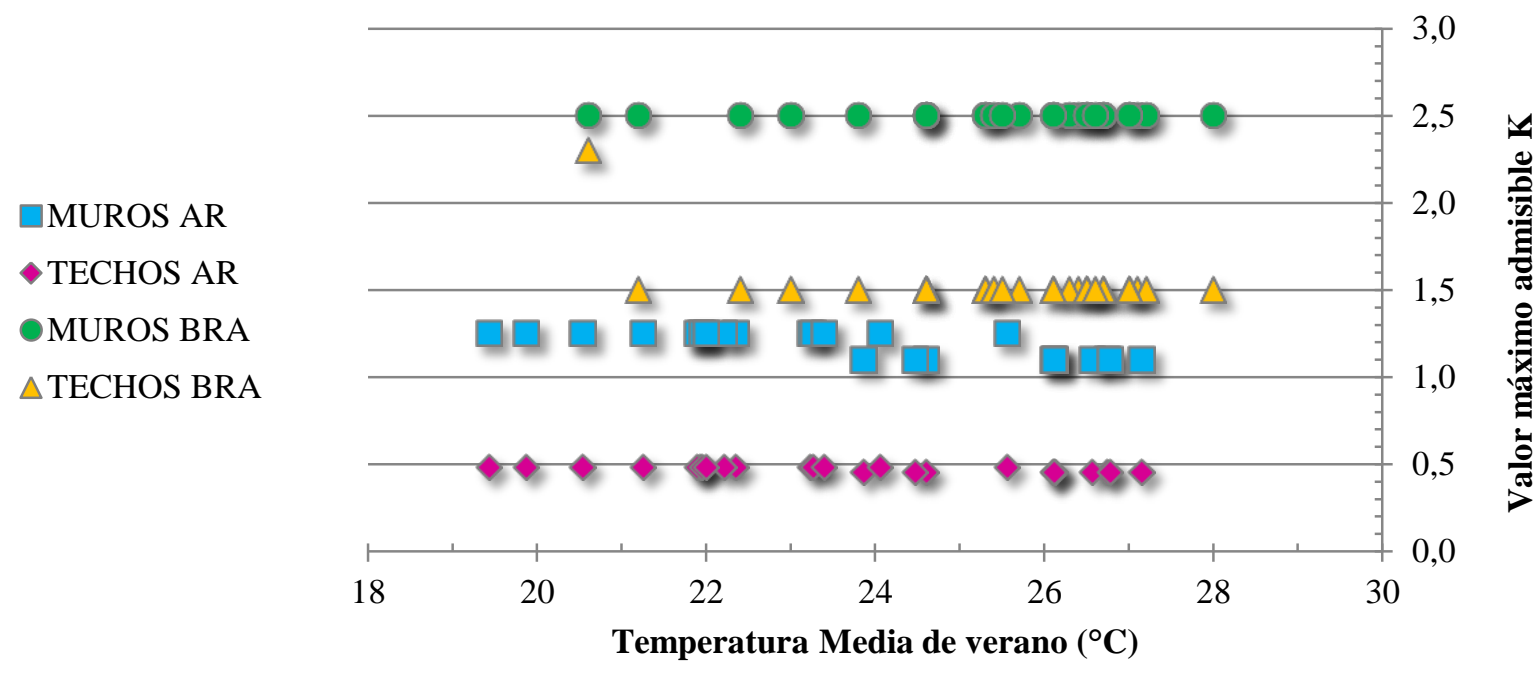

Fuente: IRAM 11603 (INSTITUTO..., 2012), NBR 15575 (ABNT, 2013) y Instituto Nacional de Metereologia do Brasil $\left(\right.$ INMET) ${ }^{6}$.

\footnotetext{
${ }^{5}$ Fuente< http: / / www.inmet.gov.br/portal/ >. ${ }^{6}$ Idem.
}

118 Reus Netto, G.; Czajkowski, J. D. 
Figura 4 - Relación entre las temperaturas medias de invierno de las localidades de Argentina y Brasil, en función de los valores de Kadm para muros y techos

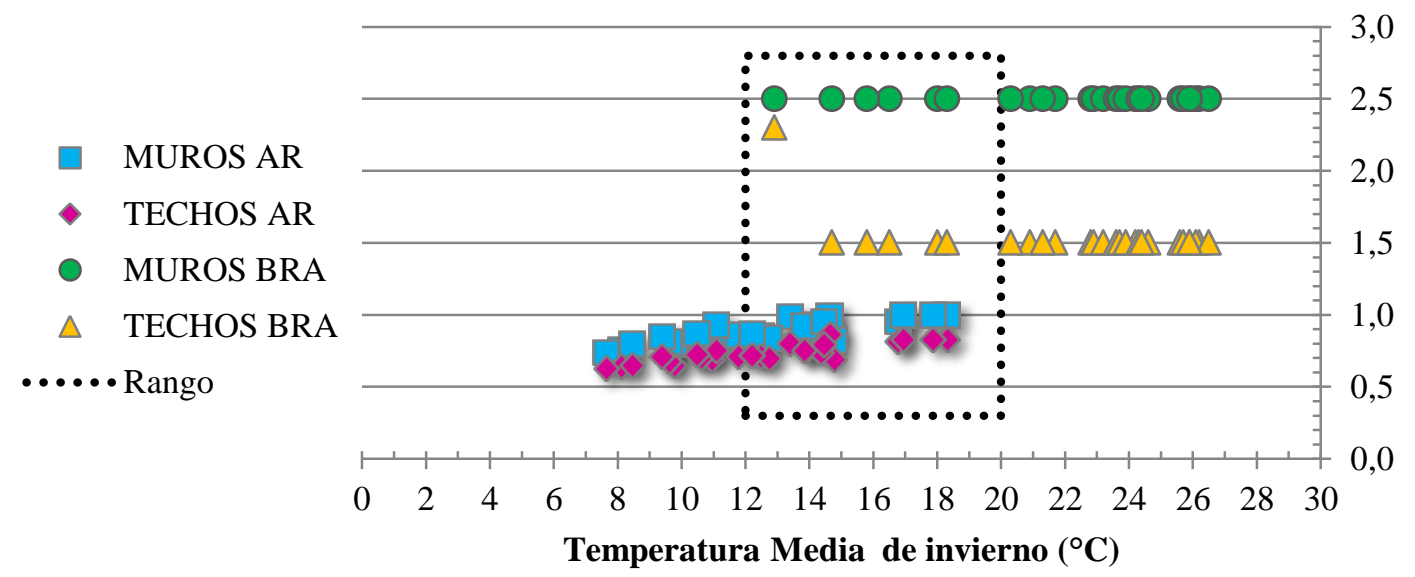

Fuente: IRAM 11603 (INSTITUTO..., 2012), NBR 15575 (ABNT, 2013) y Instituto Nacional de Metereologia do Brasil $(\text { INMET })^{7}$.

\section{Conclusiones}

Con este trabajo se pudo observar que la tendencia en garantizar el desempeño mínimo en las edificaciones, sobre todo desde el punto de vista del confort térmico es una necesidad regional.

Las normativas en Argentina han surgido bastante antes que las normas de Brasil, que aunque tardías, vienen haciendo un trayecto gradual. Se pudo observar que en Brasil, la aplicación de la NBR 15575 (ABNT, 2013) se retrasó 5 años para atender a la solicitud de los profesionales, de un mayor plazo para su implementación y a partir de eso han sido desarrollando diversos cursos $\mathrm{y}$ eventos para difundir la normativa.

Sin embargo así como en Argentina en la práctica no ha sido posible implementar en forma exhaustiva. Teniendo en cuenta la complejidad de adaptación de los profesionales a la reglamentación en la integra, en todo el territorio, y entendiendo que la aplicación de las normas es esencial para la mejora en la calidad de los edificios, se han establecido algunas pautas que contribuyan a la efectiva implementación de dichas normativas.

Primero es importante adoptar una estrategia de aplicación por etapas, disminuir parte de la rigurosidad y establecer nuevos plazos para su efectiva implementación. Es conveniente proponer un plan de acción entre organismos de autoridad y grupos de profesionales en donde se suministren cursos, charlas y reuniones promovidos por el colegio de arquitectos, ingenieros, ministerios de habitación, universidades, que busca la difusión de la normativa a los interesados, de manera que su adaptación sea gradual.

Segundo, empezar a exigir el cumplimiento de los requisitos de las normas en la etapa de aprobación del proyecto en la Municipalidad. Para ello se estima necesario la conformación de un equipo capacitado para instruir y esclarecer las dudas de los proyectistas, así como verificar los valores y sistemas de cálculo concedidos para cara obra, la finalidad es avalar y dar el permiso para la construcción, quizá con consultoría on line a cargo del estado en cada país. Es importante que después de la ejecución de la obra se pueda hacer la verificación in situ, con mediciones de temperaturas internas e superficiales y así corroborar la correcta construcción conforme detallada en los planos.

Desde el punto de vista de los criterios y requisitos exigidos por cada norma, se comprobó que las IRAM son 2 veces más exigente respecto los valores de Transmitancia Térmica $\mathrm{K}$ requeridos para muros y techo en la NBR en verano y 3,5 veces más exigentes para los valores de $\mathrm{K}$ de muros en invierno. Por ello se pretende estudiar nuevos valores para el K propuesto en la normativa brasilera y su posibilidad de aplicación. Para un segundo momento se estima estudiar la necesidad del empleo del cálculo del riesgo de condensación de manera simplificada a la NBR, así como los cálculos de pérdidas de calor por la envolvente.

Finalmente, desde el punto de vista económico y para impulsar a los profesionales, constructoras y clientes particulares, se propone que el gobierno pueda promover incentivos que busquen estimular la implementación masiva de las normativas, como

\footnotetext{
${ }^{7}$ Fuente: <http://www.inmet.gov.br/portal/>.
} 
por ejemplo que la Municipalidad reduzca por un determinado tiempo, los impuestos de la obra en función del nivel de desempeño obtenido.

Este trabajo permitió comprender los criterios y requisitos exigidos por las normas IRAM y NBR, $\mathrm{y}$ analizar las principales dificultades que enfrentan Argentina y Brasil para implementar de manera efectiva dichas normativas.

Se espera, en las próximas etapas de este trabajo, plantear sugerencias a las normativas estudiadas que contribuyan a la mejora del confort térmico edilicio, que incrementen el ahorro energético y la economía de recursos.

\section{Referencias}

AMERICAN SOCIETY FOR TESTING AND MATERIALS. ASTM C1371: standard test method for detrmination of emittance of materials near room temperature using portable emissometers. West Conshohockem 2015a.

\section{AMERICAN SOCIETY FOR TESTING AND} MATERIALS. ASTM C177: standard test method for steady-state heat flux measurements and thermal transmission properties by means of guarded-hot-plate apparatus. West Conshohocken, 2013.

\section{AMERICAN SOCIETY FOR TESTING AND} MATERIALS. ASTM E424-71: standard test methods for solar energu transmittance and reflectance (terrrestrial) of sheet materials. Wes Conshohocken, 2015b.

AMERICAN SOCIETY OF HEATING, REFRIGERATING AND AIRCONDITIONING ENGINEERS. Standard 140: standard aids in evaluating energy analysis programs. Atlanta, 2007.

ARGENTINA. Decreto 1.030. Boletín Oficial I de la Provincia de Buenos Aires, La Plata, 2010.

ARGENTINA. Ley 13.059. Legislatura de la Provincia de Buenos Aires, La Plata, 2003.

ARGENTINA. Ley 4.458. Legislatura de la Ciudad Autónoma de Buenos Aires, Buenos Aires, 2012.

\section{ASOCIACIÓN ESPAÑOLA DE}

NORMALIZACIÓN Y CERTIFICACIÓN. UNE EN 410: vidrio para la edificación: determinación de las características luminosas y solares de los acristalamientos. Madrid, 2011.

ASSOCIAÇÃO BRASILEIRA DE NORMAS TÉCNICAS. NBR 15215: iluminação natural. Rio de Janeiro, 2004.
ASSOCIAÇÃO BRASILEIRA DE NORMAS TÉCNICAS. NBR 15220: desempenho térmico de edificações. Rio de Janeiro, 2008.

\section{ASSOCIAÇÃO BRASILEIRA DE NORMAS} TÉCNICAS. NBR 15575: edificações habitacionais: desempenho. Rio de Janeiro, 2013.

BERARDI, R. et al. Reciclado Energético de la Envolvente de Vivienda Unifamiliar en La Plata, Argentina. Avances en Energías Renovables y Medio Ambiente, v. 18, p. 05.29-05.38, 2014.

CZAJKOWSKI, J. et al. Evaluación del Confort Higrotérmico Invernal en Viviendas Unifamiliares del Gran La Plata Mediante Auditorías. Avances en Energías Renovables y Medio Ambiente, v. 16, p. 5.101-5.106, 2012.

DI BERNARDO, A.; FILIPPÍN, C.; PIPA, D. Desempeño Térmico-Energético de un Prototipo Demostrativo de Vivienda de Interés Social en Córdoba, Argentina. Avances en Energías Renovables y Medio Ambiente, v. 15, p. 08.3508.42, 2011.

EVANS, J. M.; SCHILLER, S. Promoción de Eficiencia Energética en Edificios: desarrollo de la norma de etiquetado en Argentina. In:

SEMINARIO INTERNACIONAL ARQUITECTURA BIOCLIMÁTICA, SEMINARIO BIOCLIMÁTICO, 3., Azcapotzalco, 2010. Proceedings... Azcapotzalco, 2010.

GALLIPOLITI, V. et al. Evaluación del Desempeño Higrotérmico Energético de Una Vivienda Social en la Ciudad de Corrientes. Avances en Energías Renovables y Medio Ambiente, p. 05.17-05.24, 2012.

GIL, S. Eficiencia Energética: un Camino Sustentable Hacia el Autoabastecimiento. In: JORNADAS DE INTRODUCCIÓN A LA EFICIENCIA ENERGÉTICA EN ORGANISMOS PÚBLICOS, Buenos Aires, 2014. Proceedigns... Buenos Aires, 2014.

GONÇALVES, J.; DUARTE, D. Arquitetura Sustentável: uma integração entre ambiente, projeto e tecnologia em experiências de pesquisa, prática e ensino. Ambiente Construído, Porto Alegre, v. 6, n. 4, p. 51-81, out./dez. 2006.

GRIGOLETTI, G. de C.; LINCK, G. I. Análise de Comportamento Térmico de HIS Térreas Unifamiliares em Santa Maria, RS. Ambiente Construído, Porto Alegre, v. 14, n. 2, p. 109-123, abr./jun. 2014.

120 Reus Netto, G.; Czajkowski, J. D. 
INSTITUTO ARGENTINO DE

NORMALIZACIÓN Y CERTIFICACIÓN.

IRAM 11900: Etiqueta de eficiencia energética de calefacción para edificios. Clasificación según la transmitancia térmica de la envolvente. Buenos Aires, 2009.

INSTITUTO ARGENTINO DE NORMALIZACIÓN Y CERTIFICACIÓN.

IRAM 11507-1: carpinterías de obra y fachadas integrales livianas: ventanas exteriores. Requisitos básicos y clasificación. Buenos Aires, 2001a.

INSTITUTO ARGENTINO DE

NORMALIZACIÓN Y CERTIFICACIÓN.

IRAM 11507: carpinterías de obra y fachadas integrales livianas: ventanas exteriores. Buenos Aires, 2001b.

INSTITUTO ARGENTINO DE NORMALIZACIÓN Y CERTIFICACIÓN.

IRAM 11507: carpinterías de obra y fachadas integrales livianas: ventanas exteriores. Buenos Aires, 1995.

\section{INSTITUTO ARGENTINO DE}

NORMALIZACIÓN Y CERTIFICACIÓN.

IRAM 11523: carpinterías de obra: ventanas exteriores: método de ensayo de infiltración de aire. Buenos Aires, 2001c.

INSTITUTO ARGENTINO DE NORMALIZACIÓN Y CERTIFICACIÓN.

IRAM 11601: aislamiento térmico de edifícios: método de cálculo: propiedades térmicas de los componentes y elementos de construcción en régimen estacionario. Buenos Aires, 2002.

INSTITUTO ARGENTINO DE NORMALIZACIÓN Y CERTIFICACIÓN.

IRAM 11603: acondicionamiento térmico de edificios: clasificación bioambiental de la Republica Argentina. Buenos Aires, 2012.

INSTITUTO ARGENTINO DE NORMALIZACIÓN Y CERTIFICACIÓN. IRAM 11604: aislamiento térmico de edifícios: verificación de sus condiciones higrotérmicas. Ahorro de energía en calefacción: coeficiente volumétrico $\mathrm{G}$ de pérdidas de calor: cálculos y valores límites. Buenos Aires, 2001d.

INSTITUTO ARGENTINO DE NORMALIZACIÓN Y CERTIFICACIÓN.

IRAM 11605: acondicionamiento térmico de edificios: condiciones de habitabilidad en edificios: valores máximos de transmitancia térmica en cerramientos opacos. Buenos Aires, 1996.
INSTITUTO ARGENTINO DE
NORMALIZACIÓN Y CERTIFICACIÓN.

IRAM 11625: aislamiento térmico de edificios: verificación de sus condiciones higrotérmicas: verificación del riesgo de condensación de vapor de agua superficial e intersticial en los paños centrales de muros exteriores, pisos y techos de edificios en general. Buenos Aires, 2000a.

\section{INSTITUTO ARGENTINO DE}

NORMALIZACIÓN Y CERTIFICACIÓN.

IRAM 11630: aislamiento térmico de edificios. Verificación de sus condiciones higrotérmicas: verificación del riesgo de condensación de vapor de agua superficial e intersticial en puntos singulares de muros exteriores, pisos y techos de edificios en general. Buenos Aires, 2000b.

\section{INSTITUTO ARGENTINO DE} NORMALIZACIÓN Y CERTIFICACIÓN. IRAM 11659-1: aislamiento térmico de edificios: verificación de sus condiciones higrotérmicas: ahorro de energía en refrigeración: parte 1: vocabulario, definiciones, tablas y datos para determinar la carga térmica de refrigeración. Buenos Aires, 2004.

\section{INSTITUTO ARGENTINO DE}

NORMALIZACIÓN Y CERTIFICACIÓN.

IRAM 11659-2: aislamiento térmico de edificios: verificación de sus condiciones higrotérmicas: Ahorro de energía en refrigeración. Parte 2: Edificios para viviendas. Buenos Aires, 2007.

INTERNATIONAL ORGANIZATION FOR STANDARDIZATION. ISO 7726: ergonomics of the thermal environment: instruments for measuring physical quantities. Genebra, 1988.

\section{INTERNATIONAL ORGANIZATION FOR} STANDARDIZATION. ISO 8302: thermal insulation: determination of steady-state thermal resistance and related properties: guarded hot plate apparatus. Genebra, 1991.

RORIZ, M.; GHISI, E.; LAMBERTS, R. Uma Proposta de Norma Técnica Brasileira Sobre Desempenho Térmico de Habitações Populares. In: ENCONTRO NACIONAL DE CONFORTO NO AMBIENTE CONSTRUÍDO E II ENCONTRO LATINO-AMERICANO DE CONFORTO NO AMBIENTE CONSTRUÍDO, 5., Fortaleza, 1999. Anais... Fortaleza, 1999.

SILVA, A. T. et al. Comparação Entre os Processos de Implantação do Código Técnico das Edificações na Espanha e NBR 15.575/2008 Desempenho no Brasil. In: ENCONTRO NACIONAL, 6.; ENCONTRO LATINOAMERICANO SOBRE EDIFICAÇÕES E COMUNIDADES SUSTENTÁVEIS, 4., Vitória, 2011. Anais... Vitória, 2011. 


\section{Gabriela Reus Netto}

Laboratorio de Arquitectura y Hábitat Sustentable, Facultad de Arquitectura y Urbanismo | Universidad Nacional de La Plata | Calle 47, 162, La Plata | CP 1900 | Buenos Aires - Argentina | Tel.: +54 (221) 423-6587 Int. 255 | E-mail: gabi_reus@hotmail.com

Jorge Daniel Czajkowski

Laboratorio de Arquitectura y Hábitat Sustentable, Facultad de Arquitectura y Urbanismo | Universidad Nacional de La Plata |

E-mail: czajko@yahoo.com

Revista Ambiente Construído

Associação Nacional de Tecnologia do Ambiente Construído

Av. Osvaldo Aranha, $99-3^{\circ}$ andar, Centro

Porto Alegre - RS - Brasil

CEP 90035-190

Telefone: +55 (51) 3308-4084

Fax: +55 (51) 3308-4054

www.seer.ufrgs.br/ambienteconstruido

E-mail: ambienteconstruido@ufrgs.br

122 Reus Netto, G.; Czajkowski, J. D. 